\title{
The Effect of Changing the Microstructure of a Microemulsion on Chemical Reactivity
}

C. Cabaleiro-Lago, L. García-Río, P. Hervella

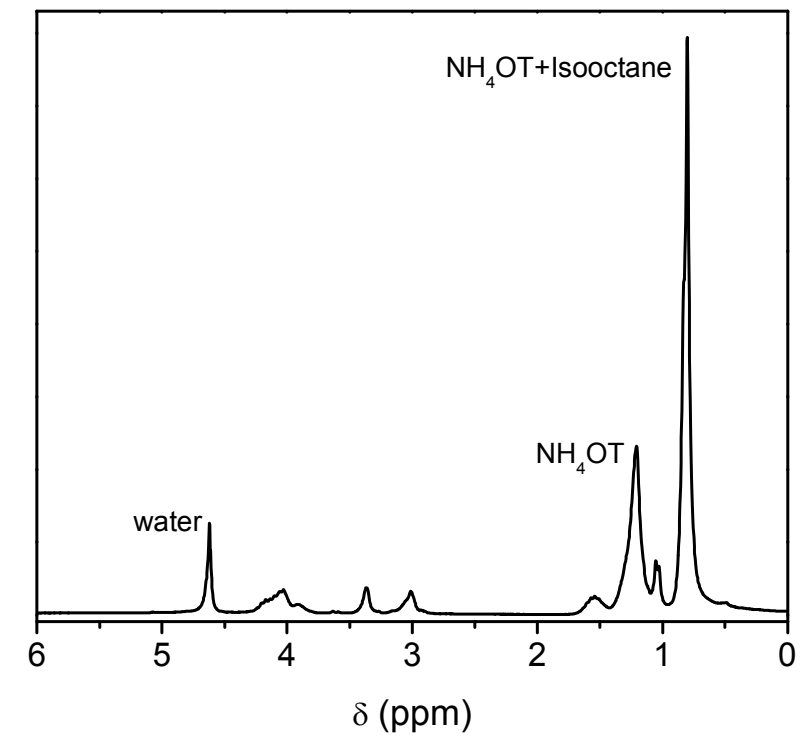

Figure S-1: ${ }^{1} \mathrm{H}-\mathrm{NMR}$ spectrum of a $\mathrm{NH}_{4} \mathrm{OT} /$ isooctane/water microemulsion (46\% water). Spectral width $2003.1 \mathrm{~Hz}$, number of points 1024 . Labels show peaks for different components. 


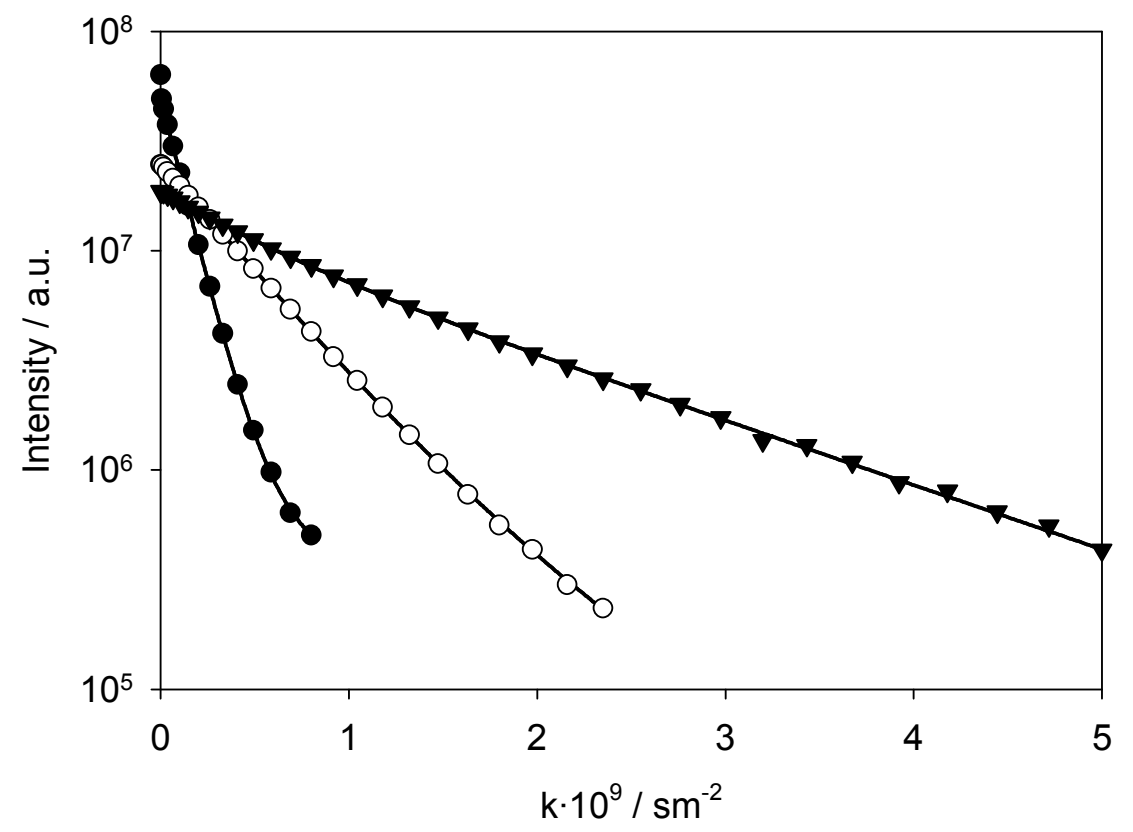

Figure S-2. Representative echo decays of $(\bullet)$ Water ( $(\circ)$ isooctane and $(\boldsymbol{\nabla}) \mathrm{NH}_{4} \mathrm{OT}$ in microemulsion. The data have been fitted to a biexponential function (the solid lines). $k$ is defined as $k=\gamma^{2} G^{2} \delta^{2}(\Delta-\delta / 3)$. 


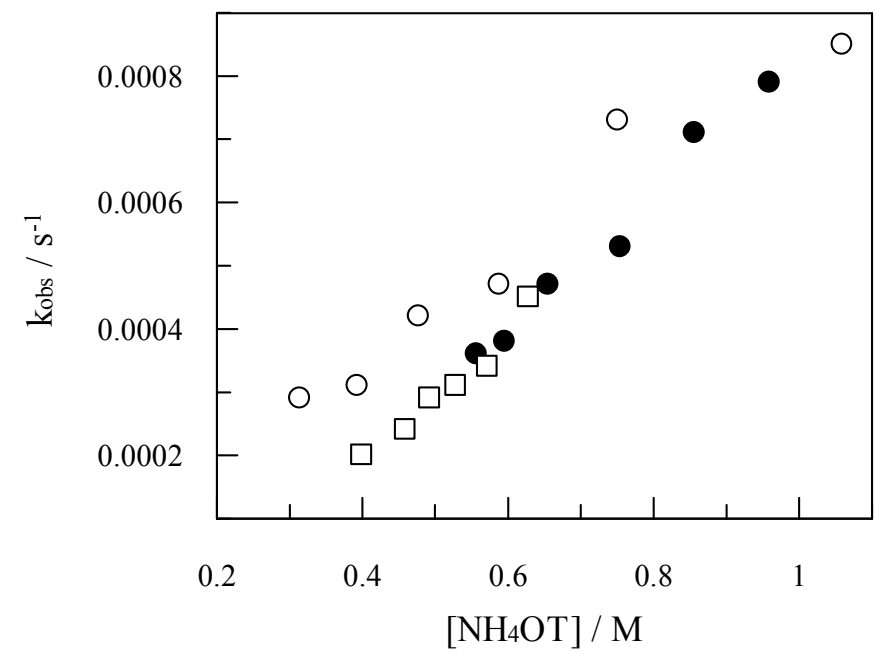

Figure S-3. Influence of the surfactant concentration on the solvolysis rate constant of $\mathrm{PhOCOCl}$ in microemulsions of water $/ \mathrm{NH} \mathrm{H}_{4} \mathrm{OT} / \mathrm{isooctane}$ at $25.0^{\circ} \mathrm{C}$. $(\circ) \mathrm{W}=5.53 ;(\bullet)$ $\mathrm{W}=25.9$ and (口) $\mathrm{W}=42.9$ 


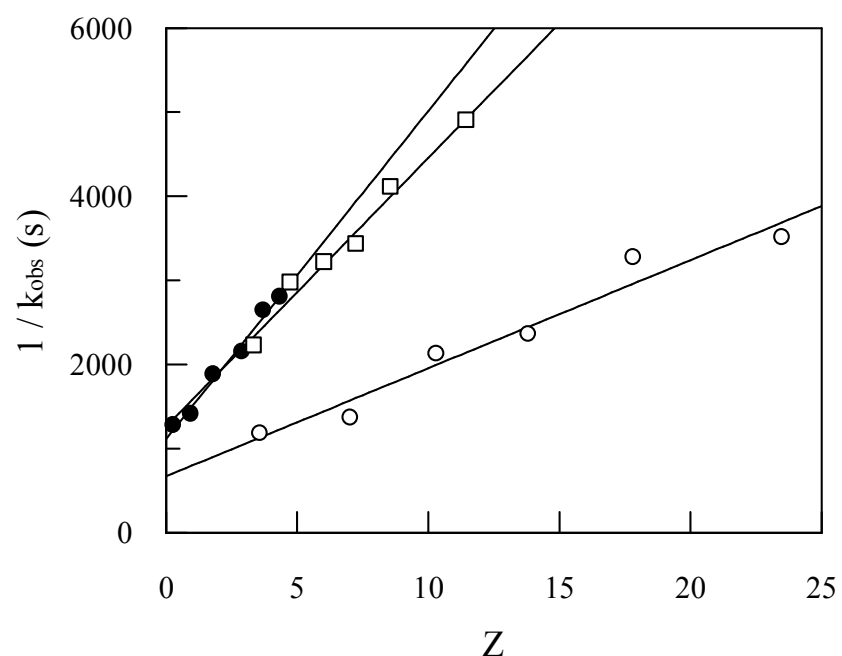

Figure S-4. Linealization of data from figure S-3 according to equation 3 (see manuscript). (०) W=5.53; (•) W=25.9 and ( $\square$ ) W=42.9. 
Table S-1. Values of the distribution constant for different substrates of the microemulsion, $K_{o i}$, for different W values.

\begin{tabular}{|c|c|c|c|c|c|c|c|c|c|c|c|c|c|}
\hline & \multicolumn{12}{|c|}{$\mathbf{W}$} & \multirow[b]{2}{*}{ Mean } \\
\hline & 2.6 & 4.0 & 5.5 & 5.8 & 9.7 & 15.0 & 20.8 & 25.9 & 40.0 & 41.8 & 54.1 & 87.2 & \\
\hline${ }_{\mathrm{DPhMeCl}}^{\mathrm{C}}$ & & & $3.8 \pm 0.4$ & & & & & $3.6 \pm 0.4$ & & & $2.5 \pm 0.3$ & $3.2 \pm 0.4$ & $3.3 \pm 0.4$ \\
\hline $4-\mathrm{MeO}$ & & $4.8 \pm 0.5$ & & $5.2 \pm 0.6$ & & $5.0 \pm 0.6$ & $5.2 \pm 0.6$ & & $5.1 \pm 0.6$ & & & & $5.1 \pm 0.6$ \\
\hline${ }_{4-\mathrm{Me}}^{\mathrm{Me}-{ }_{\mathrm{Cl}}^{\mathrm{C}}}$ & $3.2 \pm 0.3$ & & & & $2.1 \pm 0.3$ & & & $2.5 \pm 0.3$ & & & & & $2.6 \pm 0.3$ \\
\hline $\mathrm{Cl}$ & $2.3 \pm 0.3$ & & & & $2.5 \pm 0.4$ & & & $2.3 \pm 0.3$ & & & & & $2.6 \pm 0.3$ \\
\hline $4-\mathrm{CF}_{3}$ & & & $6.3 \pm 0.6$ & & & & & $4.1 \pm 0.5$ & & $5.5 \pm 0.6$ & & & $5.3 \pm 0.6$ \\
\hline$\underbrace{}_{\mathrm{PhOCOCl}}$ & & & $5.2 \pm 0.5$ & & & & & $2.8 \pm 0.6$ & & $3.9 \pm 0.5$ & & & $4.0 \pm 0.5$ \\
\hline $4-\mathrm{Cl}-\mathrm{PhOCOCl}$ & $3.2 \pm 0.3$ & & & & $2.1 \pm 0.4$ & & & $3.6 \pm 0.4$ & $3.0 \pm 0.4$ & & & & $3.0 \pm 0.4$ \\
\hline
\end{tabular}


Table S-2. Influence of $\mathrm{W}$ on the solvolysis rate constant at the interphase of $\mathrm{NH}_{4} \mathrm{OT}$-based microemulsions, $k_{i}$, for processes that take place via a dissociative mechanism. The uncertainty in the $k_{i}$ values is smaller than $10 \%$.

\begin{tabular}{|c|c|c|c|c|c|}
\hline W & $k_{i} / \mathbf{s}^{-1}$ & $\mathbf{W}$ & $k_{i} / \mathbf{s}^{-1}$ & W & $k_{i} / \mathbf{s}^{-1}$ \\
\hline 1.5 & $4.55 \times 10^{-5}$ & 1.5 & $1.34 \times 10^{-3}$ & 1.4 & $2.51 \times 10^{-4}$ \\
\hline 2.6 & $5.95 \times 10^{-3}$ & 3.1 & $3.62 \times 10^{-3}$ & 2.6 & $3.16 \times 10^{-4}$ \\
\hline 3.1 & $6.97 \times 10^{-5}$ & 4 & $7.30 \times 10^{-3}$ & 2.9 & $3.58 \times 10^{-4}$ \\
\hline 4.8 & $1.55 \times 10^{-4}$ & 4.8 & $8.39 \times 10^{-3}$ & 3.5 & $4.36 \times 10^{-4}$ \\
\hline 5.5 & $4.40 \times 10^{-4}$ & 5.8 & $1.36 \times 10^{-2}$ & 4.5 & $5.21 \times 10^{-4}$ \\
\hline 6.5 & $4.92 \times 10^{-4}$ & 6.5 & $1.75 \times 10^{-2}$ & 5.3 & $6.65 \times 10^{-4}$ \\
\hline 10.4 & $8.93 \times 10^{-4}$ & 10.4 & $3.71 \times 10^{-2}$ & 6.1 & $7.83 \times 10^{-4}$ \\
\hline 14.7 & $1.75 \times 10^{-3}$ & 14.7 & $5.57 \times 10^{-2}$ & 7.9 & $1.12 \times 10^{-3}$ \\
\hline 19.6 & $2.55 \times 10^{-3}$ & 15 & $5.94 \times 10^{-2}$ & 9.3 & $1.09 \times 10^{-3}$ \\
\hline 25.2 & $3.00 \times 10^{-3}$ & 19.6 & $7.92 \times 10^{-2}$ & 9.7 & $1.46 \times 10^{-3}$ \\
\hline 25.9 & $3.07 \times 10^{-3}$ & 20.8 & $9.85 \times 10^{-2}$ & 11.7 & $1.69 \times 10^{-3}$ \\
\hline 31.7 & $3.90 \times 10^{-3}$ & 25.2 & $1.04 \times 10^{-1}$ & 17.6 & $3.41 \times 10^{-3}$ \\
\hline 39.2 & $4.55 \times 10^{-3}$ & 31.6 & $1.21 \times 10^{-1}$ & 25 & $5.38 \times 10^{-3}$ \\
\hline 48.2 & $6.19 \times 10^{-3}$ & 39.1 & $1.41 \times 10^{-1}$ & 28.4 & $6.10 \times 10^{-3}$ \\
\hline 48.2 & $5.96 \times 10^{-3}$ & 40 & $1.79 \times 10^{-1}$ & 36.7 & $7.97 \times 10^{-3}$ \\
\hline 54.1 & $1.08 \times 10^{-2}$ & 48 & $1.68 \times 10^{-1}$ & 45 & $1.02 \times 10^{-2}$ \\
\hline 62.9 & $1.01 \times 10^{-2}$ & 58.7 & $2.11 \times 10^{-1}$ & 52.7 & $9.68 \times 10^{-3}$ \\
\hline
\end{tabular}


Table S-2. Continuation.

\begin{tabular}{|r|r|r|r|r|r|}
\hline $\mathbf{W}$ & $k_{i} / \mathbf{s}^{-1}$ & $\mathbf{W}$ & $k_{i} / \mathbf{s}^{-1}$ & $\mathbf{W}$ & $k_{i} / \mathbf{s}^{-1}$ \\
\hline 71.7 & $1.09 \times 10^{-2}$ & 67.3 & $2.40 \times 10^{-1}$ & 76.4 & $1.95 \times 10^{-2}$ \\
\hline 80.5 & $1.21 \times 10^{-2}$ & 79.4 & $2.75 \times 10^{-1}$ & 99.4 & $1.95 \times 10^{-2}$ \\
\hline 87.2 & $1.26 \times 10^{-2}$ & 104.8 & $2.99 \times 10^{-1}$ & 120.1 & $3.62 \times 10^{-2}$ \\
\hline 89.3 & $1.57 \times 10^{-2}$ & 131.8 & $2.92 \times 10^{-1}$ & 148.9 & $4.25 \times 10^{-2}$ \\
\hline 98.1 & $1.59 \times 10^{-2}$ & 134.5 & $3.05 \times 10^{-1}$ & 194.7 & $6.26 \times 10^{-2}$ \\
\hline 108 & $1.51 \times 10^{-2}$ & 149.4 & $3.18 \times 10^{-1}$ & 235.7 & $8.03 \times 10^{-2}$ \\
\hline 172 & $2.04 \times 10^{-2}$ & 202.5 & $3.38 \times 10^{-1}$ & 288.1 & $9.12 \times 10^{-2}$ \\
\hline 204 & $2.58 \times 10^{-2}$ & 356.5 & $3.49 \times 10^{-1}$ & & \\
\hline 209 & $2.48 \times 10^{-2}$ & & & & \\
\hline
\end{tabular}


Table S-3. Influence of $\mathrm{W}$ on the solvolysis rate constant at the interphase of $\mathrm{NH}_{4} \mathrm{OT}$-based microemulsions, $k_{i}$, for processes that take place via a dissociative mechanism. The uncertainty in the $k_{i}$ values is smaller than $10 \%$.

\begin{tabular}{|c|c|c|c|c|c|c|c|}
\hline $\mathbf{W}$ & $k_{i} / \mathbf{s}^{-1}$ & $\mathbf{W}$ & $k_{i} / \mathbf{s}^{-1}$ & $\mathbf{W}$ & $k_{i} / \mathbf{s}^{-1}$ & $\mathbf{W}$ & $k_{i} / \mathbf{s}^{-1}$ \\
\hline 1.1 & $2.39 \times 10^{-3}$ & 2 & $1.04 \times 10^{-2}$ & 1.5 & $2.37 \times 10^{-3}$ & 1.4 & $7.48 \times 10^{-3}$ \\
\hline 2.6 & $2.92 \times 10^{-3}$ & 4 & $7.53 \times 10^{-3}$ & 3.1 & $1.86 \times 10^{-3}$ & 2.6 & $5.95 \times 10^{-3}$ \\
\hline 6.2 & $1.19 \times 10^{-3}$ & 5.5 & $1.18 \times 10^{-2}$ & 4.8 & $1.58 \times 10^{-3}$ & 2.9 & $5.81 \times 10^{-3}$ \\
\hline 9.2 & $9.93 \times 10^{-4}$ & 8 & $4.61 \times 10^{-3}$ & 5.5 & $1.49 \times 10^{-3}$ & 4.5 & $4.26 \times 10^{-3}$ \\
\hline 9.7 & $9.89 \times 10^{-4}$ & 16 & $3.51 \times 10^{-3}$ & 6.5 & $1.45 \times 10^{-3}$ & 6.1 & $3.92 \times 10^{-3}$ \\
\hline 12.4 & $8.87 \times 10^{-4}$ & 20 & $3.86 \times 10^{-3}$ & 10.3 & $1.18 \times 10^{-3}$ & 7.9 & $3.64 \times 10^{-3}$ \\
\hline 19.8 & $8.16 \times 10^{-4}$ & 25 & $3.39 \times 10^{-3}$ & 14.6 & $1.07 \times 10^{-3}$ & 9.7 & $3.41 \times 10^{-3}$ \\
\hline 24.3 & $8.03 \times 10^{-4}$ & 25.9 & $3.46 \times 10^{-3}$ & 19.5 & $9.95 \times 10^{-4}$ & 11.7 & $3.14 \times 10^{-3}$ \\
\hline 25.9 & $1.16 \times 10^{-3}$ & 30 & $3.07 \times 10^{-3}$ & 25.1 & $9.28 \times 10^{-4}$ & 17.6 & $2.54 \times 10^{-3}$ \\
\hline 29.2 & $7.98 \times 10^{-4}$ & 35 & $2.80 \times 10^{-3}$ & 25.9 & $9.00 \times 10^{-4}$ & 25.9 & $2.46 \times 10^{-3}$ \\
\hline 34.8 & $8.09 \times 10^{-4}$ & 40 & $2.62 \times 10^{-3}$ & 31.5 & $8.68 \times 10^{-4}$ & 28.4 & $2.24 \times 10^{-3}$ \\
\hline 48.6 & $8.54 \times 10^{-4}$ & 45 & $2.73 \times 10^{-3}$ & 39.1 & $8.19 \times 10^{-4}$ & 36.7 & $2.06 \times 10^{-3}$ \\
\hline 57.3 & $1.51 \times 10^{-3}$ & 50 & $2.90 \times 10^{-3}$ & 47.9 & $7.80 \times 10^{-4}$ & 40 & $1.95 \times 10^{-3}$ \\
\hline 63 & $1.28 \times 10^{-3}$ & 60 & $3.33 \times 10^{-3}$ & 58.6 & $7.43 \times 10^{-4}$ & 45 & $1.89 \times 10^{-3}$ \\
\hline 72 & $1.32 \times 10^{-3}$ & 70 & $3.60 \times 10^{-3}$ & 62.7 & $7.94 \times 10^{-4}$ & 55.1 & $2.02 \times 10^{-3}$ \\
\hline 81 & $1.38 \times 10^{-3}$ & 80 & $2.72 \times 10^{-3}$ & 71.5 & $7.83 \times 10^{-4}$ & 86 & $2.29 \times 10^{-3}$ \\
\hline
\end{tabular}




\begin{tabular}{|r|r|r|r|r|r|r|r|}
\hline $\mathbf{W}$ & $k_{i} / \mathbf{s}^{-1}$ & $\mathbf{W}$ & $k_{i} / \mathbf{s}^{-1}$ & $\mathbf{W}$ & $k_{i} / \mathbf{s}^{-1}$ & $\mathbf{W}$ & $k_{i} / \mathbf{s}^{-1}$ \\
\hline 132 & $1.49 \times 10^{-3}$ & 90 & $2.66 \times 10^{-3}$ & 80.7 & $7.93 \times 10^{-4}$ & 276.9 & $1.45 \times 10^{-3}$ \\
\hline 173 & $2.11 \times 10^{-3}$ & 100 & $2.38 \times 10^{-3}$ & 89.3 & $7.56 \times 10^{-4}$ & & \\
\hline 185 & $2.23 \times 10^{-3}$ & 120 & $3.21 \times 10^{-3}$ & 98.8 & $7.08 \times 10^{-4}$ & & \\
\hline 193 & $1.93 \times 10^{-3}$ & 130 & $3.45 \times 10^{-3}$ & 107.1 & $7.80 \times 10^{-4}$ & & \\
\hline 285 & $2.69 \times 10^{-3}$ & 140 & $2.71 \times 10^{-3}$ & 171.4 & $8.72 \times 10^{-4}$ & & \\
\hline 324 & $3.85 \times 10^{-3}$ & 150 & $2.82 \times 10^{-3}$ & 268.1 & $1.02 \times 10^{-3}$ & & \\
\hline & & 160 & $2.77 \times 10^{-3}$ & 308.9 & $1.07 \times 10^{-3}$ & & \\
\hline & & 170 & $2.64 \times 10^{-3}$ & 342.8 & $1.13 \times 10^{-3}$ & & \\
\hline & & 190 & $2.83 \times 10^{-3}$ & 344.3 & $1.36 \times 10^{-3}$ & & \\
\hline & & 200 & $2.87 \times 10^{-3}$ & & & & \\
\hline
\end{tabular}

